\title{
Research on the Optimization Method of Virtual Enterprise's Task Scheduling Problems in Aluminum Industry
}

\author{
Erwei Yin, Fantian Zou \& Fengxing Zou \\ College of Mechatronics Engineering and Automation, National University of Defense Technology \\ Changsha 410073, China
}

Project supported by the State Key Program of National Natural Science of China (Grant No. 60634020)

\begin{abstract}
Traditional research on production scheduling in aluminum industry, aimed to certain production process, simply pursued the output as the highest aim, scheduled based on experience, so that the result of scheduling cannot reach the global optimization, and cannot realize production scheduling and resource allocation with the aim of optimal energy consumption, resulting in the waste of energy. Taken the minimal sum of energy consumption in production, transport and stock as objective function and integrated with enterprise's experiences in production, the article establishes a model of virtual enterprise's task scheduling in aluminum industry and a hybrid distributed particle swarm optimization (PSO) algorithm is proposed to solve the problem. Finally, simulation experiment is carried out using industrial data and the result shows the optimized scheduling method has obvious optimal effect on reducing scheduling time, optimizing allocation of resources and so on, and thus the energy saving and consumption reducing purpose are obtained.
\end{abstract}

Keywords: Aluminum industry, Virtual enterprise, Task scheduling, Hybrid distributed particle swarm optimization (PSO) algorithm

\section{Introduction}

Aluminum industry is metallurgical process with the highest unit comprehensive energy consumption, and the level of production scheduling for aluminum industry also has direct connection to its energy consumption in the process of production(Chen Weiqiang, Wan Hong-yan, Wu Juanni. 2009). Traditional production scheduling in aluminum industry aimed to certain industrial process and the scheduling solution obtained often cannot reach global optimization. Virtual enterprise has such characteristics as flexible structure, prompt response and so on, which cater to fast-changing market opportunities, so more and more enterprises pay attention to it(Zhao Qiang, Xiao Renbin. 2009). Usually, the master of the alliance decomposes and mergers the productive task to several subtask firstly, and then chooses appropriate cooperative enterprises to undertake corresponding subtask, and finally finishes total productive task through cooperative enterprise's joint effort(Huang Aihua, Ye Fei.).

VE task scheduling model has the characteristics such as multiple target, multiple constraints, nonlinear and so on, which is a difficult problem of NP(Gao Yang, Jaing Zibin. 2007). The article takes the optimal energy consumption in production, transport and stock which VE uses to finish productive task in aluminum industry as objective function, establishes a nonlinear mathematical planning model and a hybrid distributed particle swarm optimization (PSO) algorithm is proposed to solve the problem(HDPSO). The approach effectively uses the fast and random global search capacity of genetic algorithm (GA) and parallel distributed positive feedback mechanism of particle swarm optimization (PSO). Through discretization, it avoid redundant search in the real number range. The effect is obvious. Simulation results shows the method can reach the goal of reducing scheduling time and saving energy and reducing consumption under the premise of satisfying VE's operation requirements for aluminum industry.

\section{The description of problem and mathematical model}

\subsection{The description of problem}

Because the consumption of primary aluminum is increasing quickly and the fixed cost for producing per ton aluminum is decreasing with the expansion of the scale of aluminum plant, the scale of construction of modern aluminum plant is still growing and thus consists large aluminum production bases, so that each base can be made up of complete aluminum industrial production system including mining station, aluminum oxide plant, electrolytic aluminum plant, aluminum processing plant and so on, from bauxite to alumina, and then from alumina electrolysis to aluminum, even to the products such as Aluminum sections, planks, wires(Zhang Zhaolu, Cui Zhaojie, Zhang Luqiang. 2009). Therefore, aluminum production system may be seen as a virtual enterprise of aluminum production. Each correlative mining station, aluminum oxide plant, electrolytic aluminum plant, aluminum processing plant is the node of virtual enterprise, which consist the supply chain alliance of virtual enterprise and provide conditions for realizing the production scheduling and optimization of aluminum production system. 
Now the process of aluminum production system is properly simplified, from mining bauxite to putting the products of aluminum on sale. All the process is classified roughly and mainly includes four sections as shown in figure 1 .

In fact, the problem of virtual enterprise's production task scheduling in aluminum industry is similar to hybrid flow shop scheduling problem, but because the constraint relations between virtual enterprise's tasks are very complex, it is needed to be further extended so as to reach ideal goal of global optimization under the condition of ensuring time limit.

\subsection{The establishment of the VE task scheduling model in aluminum industry}

1) To make such assumptions about the model considering the characteristics of VE in aluminum industry:

(1) According to the difference of contract orders, the products are classified into several lots and the time that each lot is processed in different plants is known;

(2) Considering production capacity and resource conditions, each plant only processes one lot during the same period of time;

(3) VE begins to operate from 0:00

1) Symbol definition:

$N$ : the sum of lots to be processed;

$S$ : the sum of productive tasks of virtual enterprise

$M_{j}$ : the number of candidate enterprise for the productive task $j$

$N_{k}$ : the number of the lot to be arranged to be produced in the enterprise $k$

$x_{i j k}: 0-1$ variable, if the task $j$ of the lot $i$ is processed in enterprise $k$, take 1 , otherwise take 0 ;

$s_{i j k}$ : the start time for the task $j$ of the lot $i$ in the enterprise $k$

$c_{i j k}$ : the completion time for the task $j$ of the lot $i$ in the enterprise $k$

$t_{i j k h}$ : the transportation time that the task $j$ of the lot $i$ is transported to the next task's enterprise $h$ after it is processed in the enterprise $k$

$e_{i j k}$ : the energy consumption that the task $j$ of the lot $i$ is processed in the enterprise $k$

$q_{i j k h}$ : the energy consumption in transport that the task $j$ of the lot $i$ is transported to the next task's enterprise $h$ after it is processed in the enterprise $k$

$\delta\left(x_{i j k}, x_{i, j+1, h}\right): 0-1$ variable, take 1 if and only if $x_{i j k}$ and $x_{i, j+1, h}$ are 1 at the same time, otherwise take 0 ;

$K$ : stock energy consumption coefficient

$T^{*}$ : the delivery date of the products

2) Objective function

At present, the simple pursuit of maximizing enterprises' interests has become the highest productive aim of many non-ferrous metallurgical enterprises. Because of the limits of theory, method and other aspects, production scheduling solutions only involves productive fees, and most of them aim to pursue output and carry out scheduling according to their experiences. The realization of the aim of output is often at the cost of sacrificing energy consumption, the efficiency of resource use is not improved substantially and the production scheduling and resource allocation aiming to optimal energy consumption cannot be achieved, leading to the waste of energy. Therefore, aiming to the optimized energy consumption for production task, material transport and stock respectively, the article adopts the form of weighted sum to set up the objective function.

$$
\begin{aligned}
f(X) & =\sum_{i=1}^{N} \sum_{j=1}^{S} \sum_{k=1}^{M_{j}} e_{i j k} x_{i j k} \\
& +\sum_{i=1}^{N} \sum_{j=1}^{S-1} \sum_{k=1}^{M_{j}} \sum_{h=1}^{M_{j+1}} q_{i j k h} \delta\left(x_{i j k}, x_{i, j+1, h}\right) \\
& +K \times\left[T^{*}-\max \left(c_{i N}\right)\right]
\end{aligned}
$$

In the formula (1), the first term represents the energy consumption that virtual enterprise in aluminum industry finishes all the productive task; the second term represents the sum of energy consumption in transport between different productive tasks; the third term represents the stock energy consumption.

3) Constraint condition 
(1) tasks assignment

$$
\begin{gathered}
\sum_{j=1}^{M_{j}} x_{i j k}=1, \quad i \in\{1,2, \cdots N\}, j \in\{1,2, \cdots S\} \\
\sum_{j=1}^{M_{j}} N_{k}=N
\end{gathered}
$$

The formula (2) shows each productive task of each lot must be produced in one candidate enterprise; the formula (3) shows the total number of the lot which assign to each parallel enterprise is $N$.

(2) the constraints of the processing sequence

$$
\left\{\begin{array}{l}
s_{i_{1}, j, k}<s_{i_{2}, j, k} \\
i_{1}<i_{2}, \quad i_{1}, i_{2} \in\left\{1,2, \cdots, N_{k}\right\}, j \in\{1,2, \cdots, S\}
\end{array}\right.
$$

The formula (4) shows the former the lot assigned to each enterprise ranks, the earlier it is processed.

(3) the constraints of time

$$
\begin{gathered}
\left\{\begin{array}{l}
c_{i j k}+t_{i j k h} \leq s_{i, j+1, h} \\
i \in\{1,2, \cdots, N\}, j \in\{1,2, \cdots, S-1\}
\end{array}\right. \\
c_{i_{1} j} \leq s_{i_{2} j}, i_{1}<i_{2}, i \in\left\{1,2, \cdots, N_{k}\right\}, j \in\{1,2, \cdots, S\} \quad(6) \quad T^{*} \geq \max \left(c_{i N}\right) \\
s_{i j k} \geq 0, \quad i \in\{1,2, \cdots, N\}, j \in\{1,2, \cdots, S\}
\end{gathered}
$$

The formula (5) shows each task of each lot has to start working only after the lot reaches the enterprise which undertakes the productive task; the formula (6) shows, for certain enterprise, the later lot has to start working only after the former one completes; the formula (7) shows the completion time of all the productive tasks cannot be later than the delivery time, otherwise it will lead to losing credit even contract default; the formula (8) shows the start time is nonnegative.

\section{The design of HDPSO and the solutions to the problem}

Particle swarm optimization (PSO) algorithm adopted velocity-position search model. The approach initialized randomly a group of particles, each particle represents a candidate solution in the solution space, all the particles have a fitness decided by the objective function, particles fly at a fixed speed in the search space, following the particle whose fitness is optimized at present can find the global optimal particle(I.Jacob Raglend, C.Raghuveer, G.Rakesh Avinash. 2010)(Angus F.M. Huang, Stephen J.H. Yang, Minhong Wang. 2010). Particles update themselves through tracking the most optimal solution rbest found by itself and gbest found by all the swarm. While finding out the two most optimal solutions, the particles update their velocity and position according to the formula as follows:

$$
\begin{gathered}
v_{i d}(t+1)=w v_{i d}(t)+c_{1} \text { rand }_{1}()\left(\text { gbest }_{d}-x_{i d}(t)\right) \\
+c_{2} \text { rand }_{2}()\left(\text { rbest }_{i d}-x_{i d}(t)\right) \\
x_{i d}(t+1)=x_{i d}(t)+v_{i d}(t+1)
\end{gathered}
$$

In the formula (9) and (10), $v_{i d}$ shows the velocity of the particle $i$ in the space $d, x_{i d}$ shows the position of the particle $i$ in the space $d, \quad c_{1}$ and $c_{2}$ are constants, $\operatorname{rand}_{1}()$ rand ${ }_{2}()$ are random numbers evenly distributed in the interval $[0,1]$.

\subsection{The coding and decoding of particles}

Because virtual enterprise task scheduling model in aluminum industry has high complexity and multiple constraint conditions, most of the existing scheduling methods only search in the real number space and the search efficiency is very low. (Wei Ou, Fengxing Zou, Zheng Gao. 2008) proposed a code matrix method based on genetic algorithm, which made use of matrix element and position information to deal with the constraint relations between different processes effectively. The article codes and decodes particles based on the method, and the code matrix is shown in the formula (11):

$$
P_{N \times S}=\left[\begin{array}{cccc}
p_{11} & p_{12} & \cdots & p_{1 S} \\
p_{21} & p_{22} & \cdots & p_{2 S} \\
\vdots & \vdots & \vdots & \vdots \\
p_{N 1} & p_{N 2} & \cdots & p_{N S}
\end{array}\right]
$$


Each row of the matrix $P_{N \times S}$ corresponds to each lot and each row corresponds to a productive task. Matrix element $p_{i j}$ is an integer in the interval $\left(1, M_{j}\right)$. When decoding, $p_{i j}$ shows the enterprise of the task $j$ of the lot $i$.

\subsection{The discretization of PSO algorithm}

Because virtual enterprise task scheduling model in aluminum industry the article proposes is an integer programming, but basic PSO algorithm adopts search in real number space and needs to carry out integer operation, this often leads to constraint dissatisfaction or keeping away optimal solution as well as high redundancy of the approach (Chen Ailing, Yang Genke, Wu Zhiming. 2006). Aim to the problem the article carried out discrete treatment for PSO algorithm. From the formula (9) and (10) we can know, when $x_{i d}(t)$ and $v_{i d}(t+1)$ are integers, it is also ensured that $x_{i d}(t+1)$ is an integer. So only to ensure that $v_{i d}(t+1)$ is integer all the time in the evolution. The formula (9) is improved as follows:

$$
v_{i d}(t+1)=\operatorname{int}\left(w v_{i d}(t)\right)+\phi_{1}+\phi_{2}
$$

In the formula (12), when gbest $_{d} \geq x_{i d}(t), \phi_{1}$ is an integer in the interval $\left[0, c_{1}\left(\right.\right.$ gbest $\left.\left._{d}-x_{i d}(t)\right)\right]$ according to a uniform probability distribution; When gbest $_{d} \leq x_{i d}(t), \phi_{1}$ is an integer in the interval $\left[c_{1}\left(\right.\right.$ gbest $\left.\left._{d}-x_{i d}(t)\right), 0\right]$ according to a uniform probability distribution; The calculation of $\phi_{2}$ is the same as $\phi_{1}$. The PSO approach after discretization can be shown by the formula (9) and (12).

\subsection{The improvement based on inertia weight}

Basic PSO algorithm sets inertia weight as a constant value. Bigger inertia weight has a strong global convergence capability and smaller inertia weight has a strong local convergence capability. For different problems, confirming the proportionate relationship between global search capacity and local search capacity is very important. Generally, in the process of solving the problem, thresholds of iteration should choose bigger inertia weight, with the increasing the number of iterations, search space is becoming small gradually, as well as inertia weight(S.H. Nabavi-Kerizi, M.Abadi, E. Kabir. 2010). It is an effective method to design inertia weight as self-adaptability reduces linearly. The article set inertia weight as

$$
w(g)=w_{0}-\frac{g}{g_{\max }} \times c
$$

In the formula (13), $w_{0}$ is initial weight, set as the bigger value; $g$ shows the evolutionary generation; $g_{\max }$ shows the biggest evolutionary generation; $c$ shows decay factor.

\subsection{The introduction of gene}

The search efficiency of PSO algorithm is very high at the earlier stage of computation, but at the later stage, because individual differences decrease, it is easy for the approach to fall into local extreme values. Aiming to the characteristic, the article chooses suitable gene according to the differences of the scale of the problem, after all the individuals of each generation finish iterations, according to the fitness $f_{i}$ of each particle and the relationship between the maximum $f_{\max }$ and minimum $f_{\min }$, we can judge whether each particle carries out crossover operation. The rule of crossover is:

$$
\begin{aligned}
& \text { IF } \quad f_{i} \in\left(f_{\min },\left(f_{\min }+\frac{f_{\max }-f_{\min }}{M P} \times \operatorname{rand}()\right)\right) \\
& \text { Exchange } \quad p_{u} \quad p_{v} \quad u \neq v
\end{aligned}
$$

In the formula (14), $\operatorname{rand}()$ is a random number in the interval $[0,1], p_{u} 、 p_{v}$ represents arbitrary two rows of coding respectively, after crossover, the coding sequences ensures the validity of particles.

\subsection{The choice of fitness function}

Because the aim of virtual enterprise's task scheduling model in aluminum industry is to minimize the energy consumption, in order to meet the conditions that the fitness maximize when the particles optimizes, the article set the fitness function as reciprocal of $H(X)$. So the fitness of particles is:

$$
H_{f i t}(X)=1 / \min H(X)
$$

From the formula (15) we can know, when the particles search out the optimized value, the energy consumption minimizes.

\section{Simulation experience and the result analysis}

Now take the example of certain large aluminum industry group. The numbers of candidate enterprises of each 
task are 3,3,2,2 respectively. The performance index parameters of these candidate enterprises are shown in the table 1-3. The delivery time of the products is 100 days and the stock energy consumption coefficient $K=5$. Assume the population size of PSO is 30 , the largest iteration generation is 200, the initial weight is $w_{0}=1.2$, $c_{1}=1.8, c_{2}=1.8$.

In order to verify the optimized effect of the algorithm, the article did a lot of experiments. Each experiment calculates two sets of curves: one is basic PSO algorithm's optimal particle's fitness and average fitness variation curve; the other one is improved HDPSO algorithm's variation curve, of which one test result is shown in the figure 2 .

From the figure we can know, compared basic PSO algorithm with HDPSO algorithm curves, the former has obvious prematurity, the approach falls into local optimal solution early, its global search capacity is too weak and redundancy is very high; for the later, the optimized effect with the improvement of inertia weight and introduction of mutation operator is very obvious so that the global search capacity becomes strong obviously, and the discretization of the algorithm makes its search redundancy reduces greatly. As shown in the figure 3,in the experiment, the optimal scheduling solution obtained by HDPSO algorithm had obvious optimal effect on productive task assignments and scheduling. The completion time is 106 days, which meet the requirements of delivery time. The simulation result showed adopting the optimized scheduling solutions can satisfy the requirements of aluminum production and achieved the aim to save energy and reduce consumption.

\section{Conclusions}

The research shows, it has been very limited for the space of improving enterprise's comprehensive benefits through one-side improving the production capacity and control level of the production equipments, but to realize optimized scheduling, allocate productive resource reasonably, improve flexibility of production system and the utilization efficiency of equipments and reduce the consumption of manpower, water and energy are importance to improve the key competition ability of enterprise and solve resource bottleneck problem. Aiming to virtual enterprise's task assignment problem in aluminum industry, the article took the minimized energy consumption as the objective function and established nonlinear planning model. Under the constraint conditions that the delivery time of products was satisfied, the model took the energy consumption in the production, transport and stock fully into account. Aiming to the characteristics, the article proposed a new improved HDPSO algorithm. Through a lot of experiments, the simulation results showed the structure of the optimization scheduling model is reasonable and satisfy the technological requirements: HDPSO algorithm can search out the optimal energy consumption scheduling solution quickly and the application in the virtual enterprise's task scheduling model in aluminum industry is feasible. The optimization scheduling method gives references to realize the optimized scheduling of all the process in aluminum industry and energy conservation and consumption reduction.

\section{References}

Angus F.M. Huang, Stephen J.H. Yang, Minhong Wang. (2010). Improving fuzzy knowledge integration with particle swarmoptimization. Expert Systems with Applications. 37 (2010): 8770-8783.

Chen Ailing, Yang Genke, Wu Zhiming. (2006). Hybrid discrete particle swarm optimization algorithm for capacitated vehicle routing problem. Journal of Zhejiang University SCIENCE A, 2006, 7(4): 607-614.

Chen Weiqiang, Wan Hong-yan, Wu Juanni. (2009). Life Cycle Assessment of Aluminium and the Environmental Impacts of Aluminium Industry. LIGHT METALS, 2009, 5:3-10.

Gao Yang, Jaing Zibin. (2007). Using HGA to Solve Production Planning in VE. Control and Decision, 2007, 22(8):931-938.

Huang Aihua, Ye Fei. (2002). Task Allocation and Harmonizing of Virtual Enterprise. Journal of South China University of Technology (Natural Science Edition), 2002, 30(5):1-4.

I.Jacob Raglend, C.Raghuveer, G.Rakesh Avinash. (2010). Solution to profit based unit commitment problem using particle swarm optimization. Applied Soft Computing, 10(2010): 1247-1256.

S.H. Nabavi-Kerizi, M.Abadi, E. Kabir. (2010). A PSO-based Weighting method for linear combination of neural networks. Computers and Electrical Electrical Engineering, 36(2010):886-894.

Wei Ou, Fengxing Zou, Zheng Gao. (2008). Flexible Flow-shop Scheduling Approach Based on Hybrid Particle Swarm Optimization. The $20^{\text {th }}$ Chinese Control and Decision Conference, 2008: 946-951.

Zhang Zhaolu, Cui Zhaojie, Zhang Luqiang. (2009). The designing and planning of circular economy industry chain for aluminium industry. China Mining Magazine, 2009, 18(5): 25-28.

Zhao Qiang, Xiao Renbin. (2009). Task Scheduling of Virtual Enterprise Based on Multi-Agent Technology. Journal of South China University of Technology (Natural Science Edition), 2009, 37(2):20-24. 
Table 1. the production schedule of candidate enterprise

\begin{tabular}{ccccccccccc}
\hline \multirow{2}{*}{ lot } & \multicolumn{3}{c}{ Task 1 } & \multicolumn{3}{c}{ Task 2 } & \multicolumn{3}{c}{ Task 3 } & \multicolumn{2}{c}{ Task 4 } \\
& $\mathrm{E}_{11}$ & $\mathrm{E}_{12}$ & $\mathrm{E}_{13}$ & $\mathrm{E}_{21}$ & $\mathrm{E}_{22}$ & $\mathrm{E}_{23}$ & $\mathrm{E}_{31}$ & $\mathrm{E}_{32}$ & $\mathrm{E}_{41}$ & $\mathrm{E}_{42}$ \\
\hline 1 & 5 & 6 & 3 & 8 & 9 & 13 & 10 & 6 & 5 & 6 \\
2 & 6 & 6 & 5 & 14 & 14 & 10 & 8 & 10 & 6 & 6 \\
3 & 5 & 4 & 3 & 13 & 13 & 12 & 9 & 10 & 7 & 7 \\
4 & 6 & 5 & 5 & 13 & 10 & 11 & 7 & 8 & 7 & 5 \\
5 & 4 & 6 & 5 & 13 & 14 & 12 & 10 & 10 & 5 & 6 \\
6 & 4 & 4 & 4 & 14 & 11 & 12 & 7 & 9 & 5 & 5 \\
7 & 6 & 5 & 5 & 12 & 13 & 14 & 7 & 6 & 5 & 5 \\
8 & 6 & 5 & 5 & 13 & 13 & 13 & 8 & 10 & 6 & 6 \\
9 & 5 & 5 & 4 & 14 & 11 & 11 & 9 & 10 & 5 & 6 \\
10 & 5 & 6 & 6 & 12 & 14 & 14 & 8 & 18 & 6 & 5 \\
11 & 6 & 6 & 5 & 10 & 9 & 12 & 10 & 7 & 5 & 7 \\
12 & 5 & 6 & 5 & 13 & 14 & 11 & 7 & 8 & 7 & 6 \\
\hline
\end{tabular}

Table 2. the energy consumption of candidate enterprise in the production

\begin{tabular}{ccccccccccc}
\hline \multirow{2}{*}{ lot } & \multicolumn{3}{c}{ Task 1 } & \multicolumn{3}{c}{ Task 2 } & \multicolumn{3}{c}{ Task 3 } & \multicolumn{2}{c}{ Task 4 } \\
& $\mathrm{E}_{11}$ & $\mathrm{E}_{12}$ & $\mathrm{E}_{13}$ & $\mathrm{E}_{21}$ & $\mathrm{E}_{22}$ & $\mathrm{E}_{23}$ & $\mathrm{E}_{31}$ & $\mathrm{E}_{32}$ & $\mathrm{E}_{41}$ & $\mathrm{E}_{42}$ \\
\hline 1 & 7.1 & 8.3 & 3.2 & 12.0 & 13.5 & 17.5 & 24.5 & 17.7 & 3.8 & 4.5 \\
2 & 8.2 & 10.2 & 9.5 & 14.0 & 18.0 & 12.5 & 20.5 & 24.2 & 4.6 & 5.7 \\
3 & 8.4 & 7.9 & 6.5 & 15.5 & 17.0 & 16.5 & 22.6 & 23.2 & 6.0 & 5.6 \\
4 & 10.1 & 8.2 & 8.9 & 17.0 & 13.0 & 14.5 & 19.5 & 18.9 & 5.8 & 4.3 \\
5 & 7.8 & 10.5 & 9.1 & 16.5 & 18.0 & 15.5 & 24.5 & 23.7 & 3.9 & 5.0 \\
6 & 6.3 & 6.9 & 5.8 & 17.5 & 14.5 & 15.5 & 20.8 & 22.4 & 4.9 & 4.1 \\
7 & 9.9 & 8.7 & 8.4 & 15.0 & 17.0 & 18.0 & 19.6 & 17.9 & 3.5 & 4.5 \\
8 & 10.5 & 7.2 & 8.6 & 16.5 & 16.0 & 17.0 & 22.2 & 24.1 & 5.6 & 5.2 \\
9 & 8.5 & 8.7 & 7.9 & 18.0 & 14.5 & 12.0 & 23.4 & 24.2 & 4.8 & 5.1 \\
10 & 7.8 & 8.0 & 8.5 & 16.0 & 17.5 & 18.0 & 18.7 & 18.5 & 5.5 & 4.5 \\
11 & 8.6 & 10.4 & 7.9 & 14.5 & 13.0 & 15.0 & 24.0 & 19.9 & 4.4 & 5.8 \\
12 & 8.6 & 10.8 & 8.3 & 16.0 & 17.5 & 12.5 & 20.6 & 22.5 & 5.2 & 4.0 \\
\hline
\end{tabular}

Table 3. The candidate enterprise's energy consumption in transport and schedule

\begin{tabular}{ccccccc}
\hline $\begin{array}{c}\text { candidate } \\
\text { enterprise }\end{array}$ & \multicolumn{2}{c}{ energy consumption in transport } & \multicolumn{4}{c}{ Transport time } \\
& $\mathrm{E}_{\mathrm{i}+1,1}$ & $\mathrm{E}_{\mathrm{i}+1,2}$ & $\mathrm{E}_{\mathrm{i}+1,3}$ & $\mathrm{E}_{\mathrm{i}+1,1}$ & $\mathrm{E}_{\mathrm{i}+1,2}$ & $\mathrm{E}_{\mathrm{i}+1,3}$ \\
\hline $\mathrm{E}_{11}$ & 1.3 & 1.0 & 0.6 & 2.5 & 2.0 & 1.5 \\
$\mathrm{E}_{12}$ & 0.6 & 0.9 & 1.2 & 1.5 & 2.5 & 3.0 \\
$\mathrm{E}_{13}$ & 1.4 & 1.1 & 0.9 & 3.0 & 2.5 & 2.5 \\
$\mathrm{E}_{21}$ & 3.2 & 2.1 & & 3.0 & 2.5 & \\
$\mathrm{E}_{22}$ & 3.0 & 3.5 & & 2.5 & 1.5 & \\
$\mathrm{E}_{23}$ & 2.5 & 4.0 & & 2.0 & 2.5 & \\
$\mathrm{E}_{31}$ & 1.2 & 1.0 & & 1.5 & 1.0 & \\
$\mathrm{E}_{32}$ & 2.2 & 2.4 & & 2.5 & 2.0 & \\
\hline
\end{tabular}




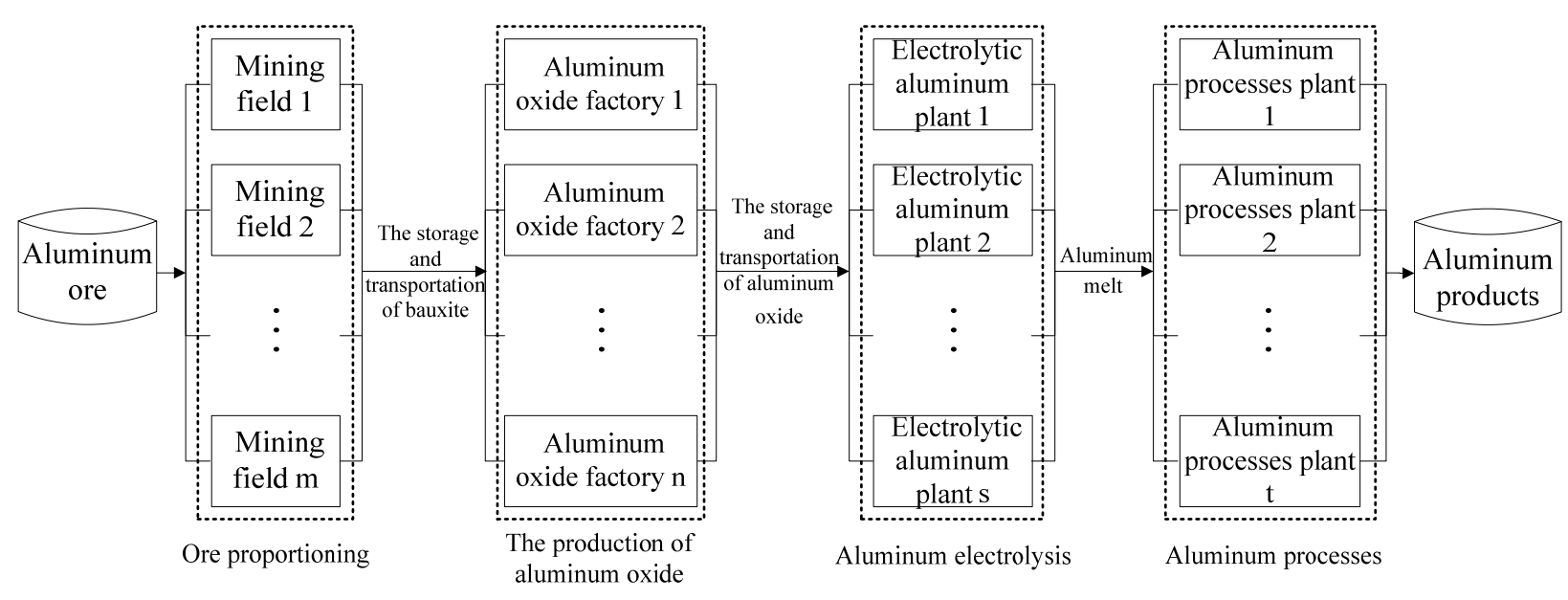

Figure 1. The production process flow chart of Aluminum industrial system

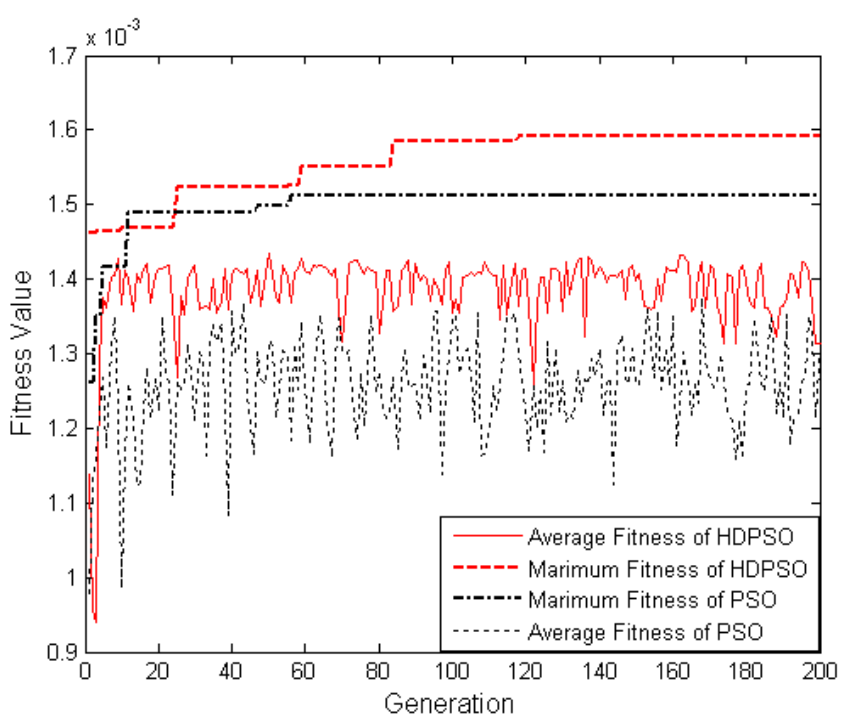

Figure 2. Particle's fitness maximum and average convergence curve

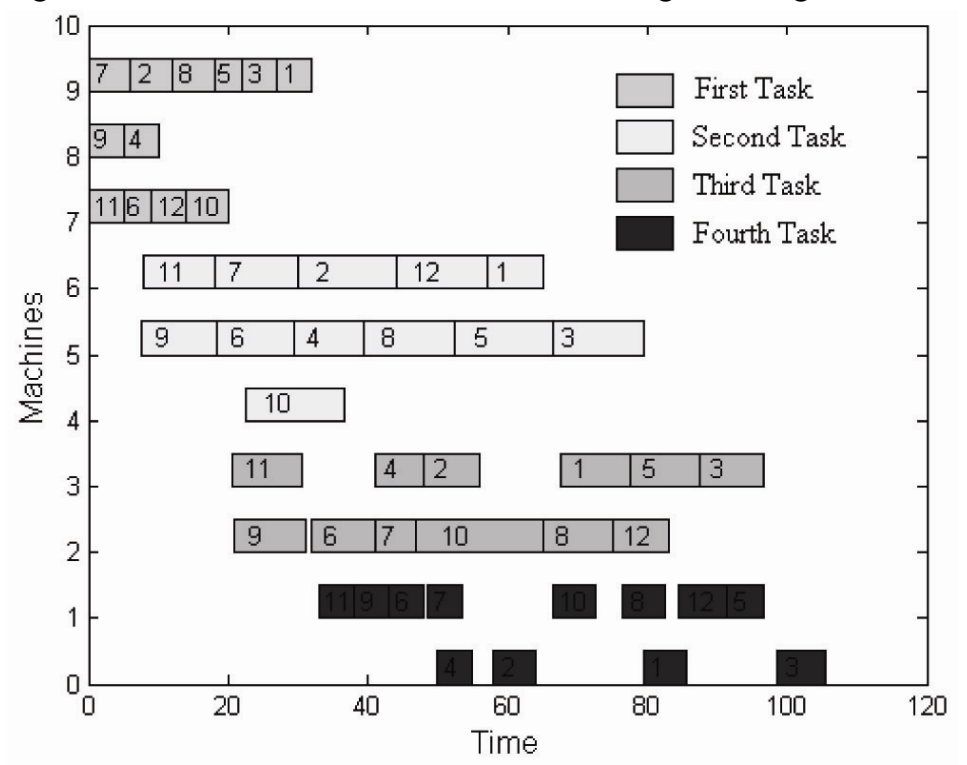

Figure 3. The Gantt chart of scheduling solutions through HDPSO approach 